Décadrages Décadrages

cınéma, à travers champs Cinéma, à travers champs

34-36 | 2017

Cinéma de re-montage

\title{
Le re-montage dans le cinéma soviétique
}

\section{François Albera}

\section{(2) OpenEdition}

\section{Journals}

Édition électronique

URL : https://journals.openedition.org/decadrages/1021

DOI : 10.4000/decadrages. 1021

ISSN : 2297-5977

\section{Éditeur}

Association Décadrages

Édition imprimée

Date de publication : 1 mai 2017

Pagination : 11-25

ISBN : 978-2-9700963-3-7

ISSN : 2235-7823

Référence électronique

François Albera, «Le re-montage dans le cinéma soviétique », Décadrages [En ligne], 34-36 | 2017, mis en ligne le 19 août 2019, consulté le 03 avril 2022. URL : http://journals.openedition.org/decadrages/ 1021 ; DOI : https://doi.org/10.4000/decadrages.1021

® Décadrages 


\section{Le re-montage dans le cinéma soviétique}

LA PRATIQUE DU «FILM DE MONTAGE», si l'on exclut sa version «naturelle» résultant de l'assemblage des vues dans un programme telle que l'inaugurent les premières séances Lumière, Edison, Skladanowsky, etc. selon une thématique (ferroviaire, géographique, historique, religieuse, etc.) et qui se poursuit dans les années I9Io et 1920 et au-delà ${ }^{1}$, a une origine marquée dans l'URSS des années 1920 avec des cinéastes comme Koulechov, Vertov, Choub notamment qui lui donnent une dimension discursive. Tous trois pratiquent ce qu'on appelle en russe alors le «re-montage» (pérémontaj).

Le mot désigne d'abord une pratique ordinaire dans tous les pays, c'est-à-dire celle de la conformation des films étrangers à la distribution nationale: montage des intertitres dans la langue locale, réduction éventuelle de la durée ou modification du rythme des films selon des standards nationaux (les versions étrangères des films muets différaient souvent d'un pays à l'autre sous de nombreux aspects). En URSS, le département de «re-montage» prit toutefois une importance particulière en raison de la nécessité de se réapproprier les actualités comme les films de fiction venus de l'étranger afin d'en donner une version compatible avec l'idéologie révolutionnaire soviétique. Il est évident quaucun film étranger entre I9I7 et I924 (date de la reconnaissance de l'URSS par plusieurs pays dont les Etats-Unis, la France, l’Allemagne) - et, par la suite, dans la majorité des cas - ne donnait dans ses actualités ni ses films documentaires ou de fiction une vision de l'URSS qui lui soit favorable. La censure de certaines images, les déplacements d'images pour leur faire signifier autre chose et le commentaire verbal des titres étaient donc d'une grande importance - s'ajoutant à l'intervention des bonimenteurs lors des séances - avant que la production soviétique puisse redémarrer et occuper les écrans. Viktor Chklovski, par ailleurs théoricien de l’Opoïaz, membre du LEF et scénariste, évoque, dans un article de 1926 resté inédit intitulé «Le ciné-montage pour la censure» ${ }^{2}$, la nécessité
1 Notamment à l'UFA où l'on assemble des vues sur des thèmes animaliers, d'activités humaines, géographiques (cf. Der Auge der Welt, i928), démarche que poursuivront d'une certaine manière les production Disney dans les années I950 avec des séries comme Le Désert vivant.

2 V. Chklovski, Textes sur le cinéma, Lausanne, L'Age d'Homme, 20II, pp.269274 (sous le titre «Le montage pour la censure»). 
3 «[Extraits de l'histoire des kinoks]». Sténogramme d'une intervention de Vertov à des ateliers de formation, daté du 2i février I929. de ce remaniement des films étrangers: «le film allemand, américain et français est parfaitement anti-démocratique, sans même parler de conscience de classe du prolétariat. C'est de la propagande consciente, passée par le filtre du réalisateur, le diktat du studio et celui du comptoir de distribution. Voilà pourquoi tous les films en URSS sont soumis au remontage». Il donne cependant - ayant eu à pratiquer ces ajustements - un certain nombre de préceptes à respecter car, dit-il, «il arrive que le matériau se rebiffe»; selon lui, «il faut modifier le film sans violence, au lieu de l'octobriser», ce qui lui fait perdre toute logique et toute crédibilité.

Il se trouve que la plus fameuse cinéaste soviétique ayant attaché son nom au film «de montage», Esfir Choub, commença sa carrière dans le re-montage de films étrangers : entre I922 et I925 environ, 200 films passèrent sous ses yeux et entre ses mains et ses ciseaux (on connaît l'exemple des deux épisodes du Doktor Mabuse (1922) de Fritz Lang - Dr Mabuse der Spieler et Dr Mabuse Inferno - qui duraient ensemble environ 5 heures et qu'elle eut à condenser en un seul film intitulé La Pourriture dorée (I924), ce qui fut aussi l'occasion d'initier Eisenstein au montage).

Le re-montage, en outre, s'effectuait également à l'endroit des films soviétiques soit pour les «corriger» en fonction d'exigences circonstancielles au moment de leur sortie, soit pour les adapter à une utilisation ultérieure ou pour l'exportation - opérations qui pouvaient être effectuées par la représentation commerciale à l'étranger. Ainsi Vertov déplore-t-il que lorsque sa Onzième Année (1928) obtint la possibilité d'être vendue à l'étranger:

«Une situation curieuse se produisit [...]. [...] le film atterrit dans la représentation commerciale, où le camarade Kisman et sa femme le visionnèrent en famille, décidèrent que le film était médiocre et se mirent à son re-montage. Ils pensèrent que La Onzième Année n’apportait pas suffisamment sur le plan cinématographique. Tseïtline, qui se trouvait là, demanda à voir le film, à quoi on répondit qu’il était incompréhensible et qu'il nécessitait un remontage. De fait, il fut découpé en morceaux. Lorsque la VUFKU l'apprit, nous réussîmes à faire en sorte que le film ne soit pas projeté dans sa version revue par les distributeurs.» ${ }^{3}$

Mais l'art du re-montage s'élargit en URSS au-delà de ces mises en conformité et adaptations - techniques, commerciales ou idéologiques -, il porte sur la recherche de nouveaux «effets » par l'utilisation de matériaux déjà filmés. Il ne s'agit plus seulement de rassembler et 
unifier divers matériaux, ni même de les "corriger», mais de les ordonner dans la perspective d'un nouveau montage. Ainsi Vertov, engagé en mai ı9ı8 par Mikhaïl Koltsov, responsable du Comité du cinéma (Kino-komitet) auprès du Commissariat du peuple à l'Instruction publique (Narkompros), pour y rédiger les cartons (intertitres) des bandes d'actualités, devenu rapidement responsable du département, et, à la fin de l’année, chargé du Kino-nédélia (La Semaine cinématographique), réalise, dès l'année suivante, un «documentaire historique» de $2 \mathrm{~h} 30$ (I2 parties), L'Anniversaire de la révolution (I919) qui reprend les «sujets» hebdomadaires qu'il avait traités au jour le jour dans une synthèse plus ample. Il réitère cette opération en I92I-I922 avec L'Histoire de la guerre civile en $\mathrm{i} 3$ bobines tout en continuant de sacrifier à la logique à court terme des actualités (43 éditions du Kino-nédélia, 23 numéros de la KinoPravda, 57 du Goskino-kalendar entre 1922 et I925). Par la suite, dans les longs métrages documentaires qu'il réalise, il n'est pas rare qu'il réinsère des sujets tournés antérieurement pour ces actualités. Trois Chants sur Lénine (1934) est un bon exemple de cette démarche, qui reprend plusieurs Kino-Pravda de 1924-I925 consacrés au dirigeant bolchévik. Et dans tous ses films à partir d'En avant Soviet! (1926) (La Onzième Année, La Sixième Partie du monde, 1926, L’Homme à la caméra, 1929, Berceuse, I937...), il donne forme à des prises de vue qu'il a dirigées voire réalisées, comme à d'autres qu'il a commandées à différents opérateurs sans se rendre sur place (c'est particulièrement le cas dans La Sixième Partie du monde). Il se donne, en effet, une fonction de coordinateur, d'organisateur au sein d'un collectif - ce qui définit une place particulière au réalisateur et fait du collectif (le Soviet des Trois puis les kinoks) «l'auteur» des films ${ }^{4}$. Le montage étant la prérogative de cet organisateur des matériaux devient le moment clé de leur mise en forme et de la construction sémantique. Vertov se trouve là en proximité avec la doctrine constructiviste dans la plupart des pratiques artistiques (notamment la photographie, le photomontage) et, en particulier, avec la conception que se faisait Serguéi Trétiakov du rôle de l'écrivain: recueillir des témoignages, des rapports de correspondants ouvriers et paysans venant de tout le pays et les organiser pour la publication ${ }^{5}$.

Cependant, on l'a dit, la monteuse Esfir Choub se distingua particulièrement à cette époque en réalisant des films de montage à partir de matériaux filmés préexistants mais de nature archivistique et dont elle
4 Ce positionnement (qui est l'un des aspects que revendiquent à leur tour Godard et Gorin au sein du Groupe DzigaVertov) se retourna contre Vertov dans les années I930-I940 où on lui dénia le statut d'auteur de ses films en le réduisant à une fonction technique. Paradoxalement, lui qui avait lutté pour l'abolition de la notion même d'art, de créateur, etc. dut revendiquer la dimension artistique de son travail et un statut de créateur.

5 Voir S. Trétiakov, Dans le Front gauche de l'art, Paris, Maspéro, I977. 
n’avait tourné ni commandité elle-même aucune image. Dans ses trois longs métrages de compilation, La Chute de la dynastie des Romanov (Padenie dinastii Romanovykh, 1927) - sur la Révolution de Février I917 -, Le Grand Chemin (Veliki put', I927) - sur la Révolution d'Octobre I9I7 -, Léon Tolstoï et la Russie des Romanov (1927, non conservé), il s'agissait

La Chute de la dynastie des Romanov (Esfir Choub, 1927)
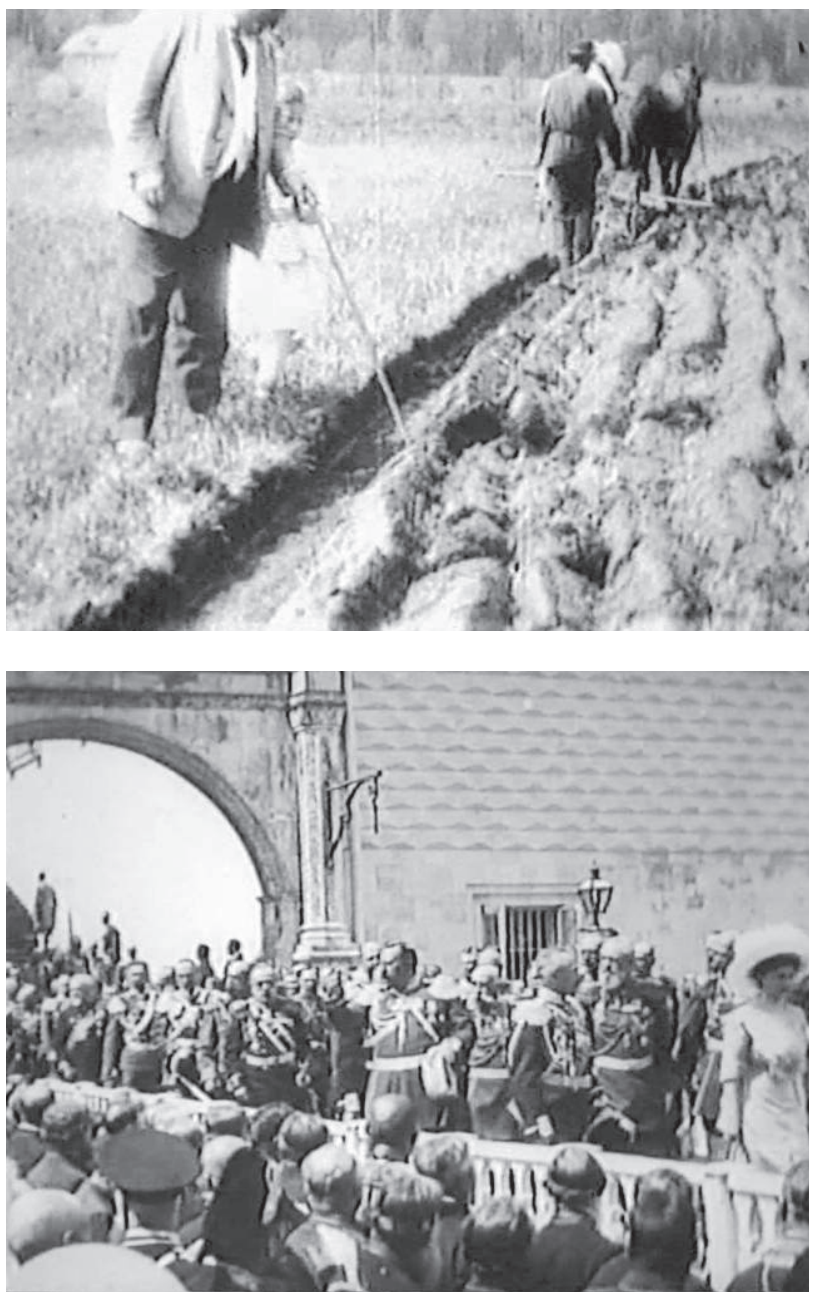

Dans l'ancien régime. Un propriétaire terrien et sa petite fille dans le sillon qu'a tracé le paysan à leur service. L'homme mesure la profondeur de sa canne. La petite fille gambade sur les mottes.
1913. Le défilé des notables durant les fêtes du tricentenaire de la dynastie des Romanov. Un gouverneur interpelle un spectateur qui ne s'est pas découvert. 
pour elle de retravailler des corpus d'actualités issues de maisons de production russes de l'époque tsariste et de maisons étrangères (françaises - comme Pathé, Gaumont -, allemandes, etc.) ou de films privés, puis des actualités du Comité Skobelev, enfin des actualités soviétiques. Sa tâche fut pionnière dans la mesure où elle eut à mener des recherches visant
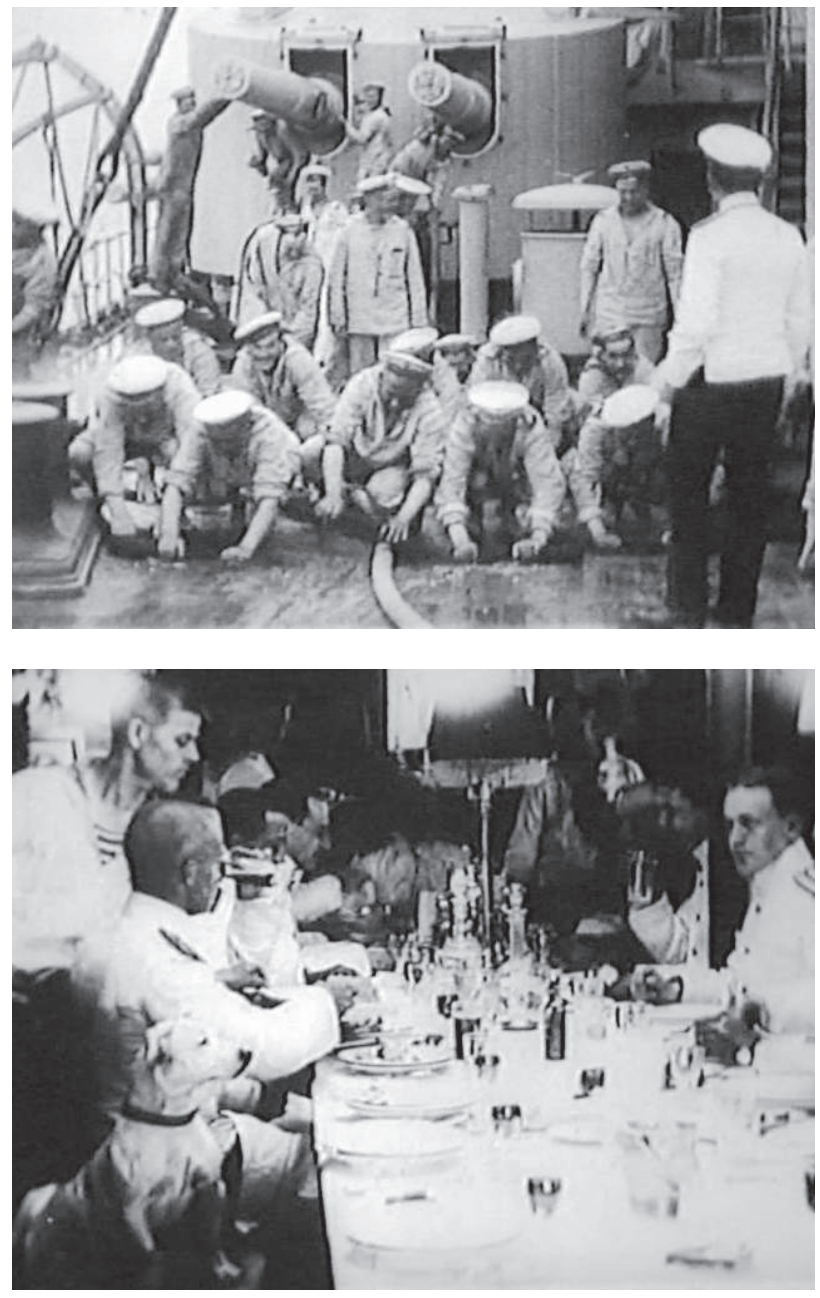

Sur un bateau de guerre impérial. Les marins lavent le pont.

Pendant ce temps les officiers déjeunent. Un chien a pris place à table. 
à localiser, identifier des archives et des documents qui n'étaient nulle part conservés en bonne et due forme, répertoriés, catalogués, suivant la trace de certains jusqu'aux Etats-Unis où ils avaient été envoyés, et à insister pour que l'URSS les récupérât ou achetât tout document sur les années ı9ıo en Russie, à établir ces sources (elle interrogea à cette
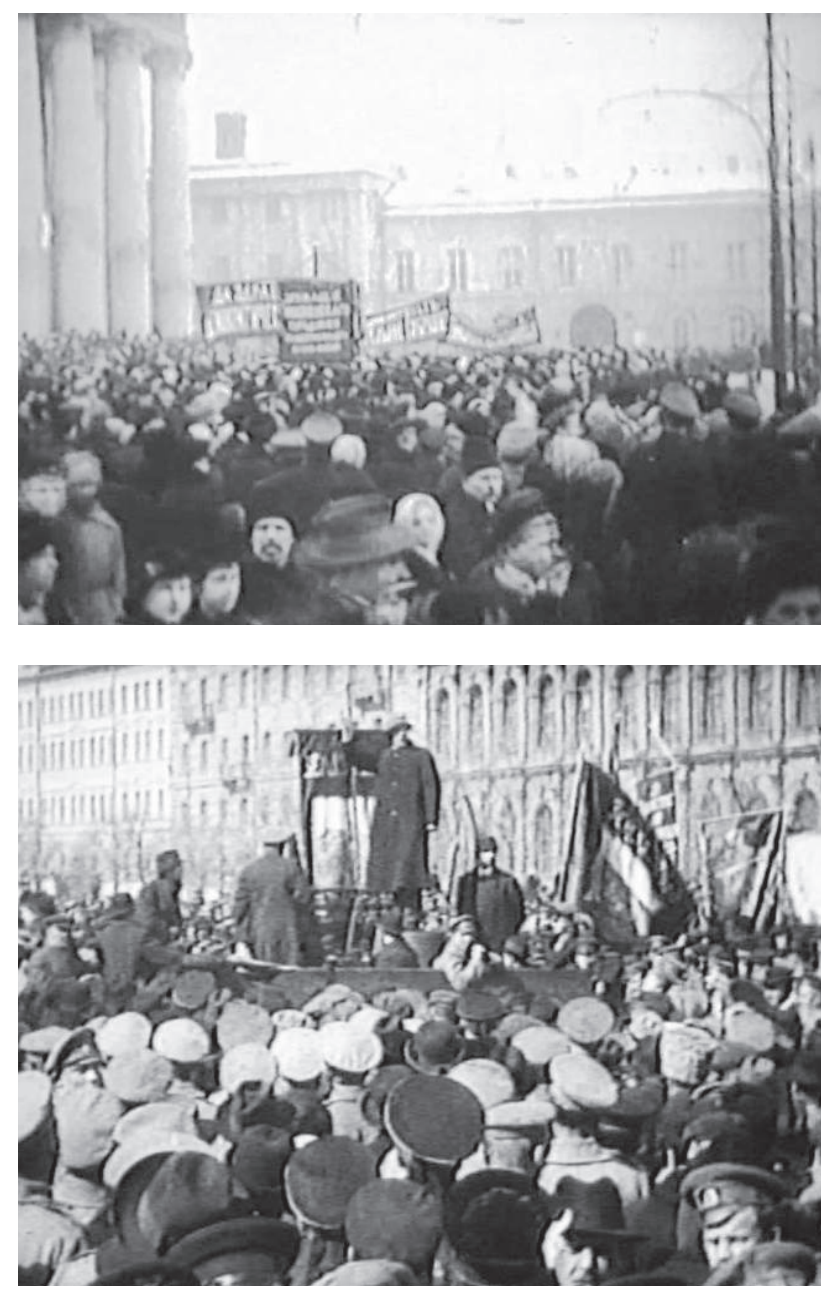

Février 1917. Les masses populaires envahissent les rues et les places jusqu'ici ordonnées strictement en fonction des hiérarchies sociales.

Février 1917. Un orateur révolutionnaire harangue la foule. 
fin les opérateurs des vues quand ils étaient accessibles $\left.{ }^{6}\right)$. Elle est donc une archiviste tout autant qu'une monteuse et fit prendre conscience de l'importance de ces matériaux filmés pour l'histoire. C'est sur cette base qu'elle entreprit ses montages qui répondent à une construction rigoureuse fondée sur une intelligence du contenu des vues. Le choix des fragments, dont souvent la longueur est respectée, les mises en rapports, en contrastes même, ne sont ainsi jamais dans ses films de brutales oppositions rhétoriques du type «riche vs pauvre», «patrons vs ouvriers»; elles prennent appui sur un détail prégnant de la vue, un événement même bénin mais signifiant qui est contenu dans l'extrait ou le fragment retenu. Ainsi peut-on relever que la scène du défilé des notables lors des festivités de 1913 du $300^{\circ}$ anniversaire des Romanov, où l'on voit un général ventripotent intimer à un spectateur qu'on ne voit pas de se découvrir, cette scène que Chris Marker a repris dans Le Tombeau d'Alexandre (I992) en arrêtant l'image, entourant le personnage d'un trait de couleur, commentant longuement la scène et sa possible signification, figure dans La Chute de la dynastie des Romanov (où Marker l'a sans doute trouvée) sans aucune intervention de soulignement et d'emphase interprétative. Par contre, elle a été choisie soigneusement pour cet «accent» comme on peut le comprendre en voyant toute une série d'autres vues de l'événement (reprises sans distinction dans un ensemble d'émissions réalisées en I990 pour le BFI). Et cet accent n’a pas échappé aux spectateurs, en tout cas à Chklovski qui le relève dans un article de $1927^{7}$.

Contrairement à Vertov, pour qui «la primitive Dynastie» n'est «absolument pas montée» ${ }^{8}$, Kouléchov, qui était passé lui-même au début de sa carrière soviétique par les actualités et revint par la suite au documentaire, voyait dans le film de Choub un remarquable travail de montage. Pourquoi? Jusqu'à présent, dit-il, les films «non joués» avaient un défaut majeur, celui d'avoir «un montage incorrect» car «subjectif et artistique», voire «expressionniste»: le montage n'y est pas «au service du matériau», ne vise pas à le mettre en valeur mais est «le lieu d'une création personnelle pour le monteur». Ces films se transforment, poursuitil, «en une combinaison d'exclamations ronflantes ou en un enchaînement de cadres symboliques ${ }^{9}$. Grâce à un montage considéré comme un «moyen d'expression et d'organisation du matériau révélant son essence thématique», Choub représente une étape nouvelle dans le documentaire soviétique ${ }^{10}$.
6 Voir Jay Leyda, Kino. Histoire du cinéma russe et soviétique, Lausanne, L’Age d'Homme, I976, p. 260 qui s'appuie sur des articles et entretiens de Choub parus dans la presse cinématographique soviétiques des années I920-I930.

7 «[... ce film est nôtre parce que la monteuse, en confrontant les cadres, nous montre Nicolas Romanov en tsar nuisible et de petite envergure. En tyran raté. / A l'occasion du tricentenaire de la dynastie des Romanov, des gens vêtus d'or sortent du Kremlin. / Parmi eux se trouve le gouverneur. Il menace de son poing la foule, tout en criant, visiblement: « Découvrez-vous!〉. Nicolas avance à petits pas, d'une démarche embarrassée.» ( $P$ Pour l'anniversaire de février. Un filmdocument», Kino, n ${ }^{\circ}$ II, I2 mars 1927, traduit dans V. Chklovski, Textes sur le cinéma, op. cit., p. 199). On a commenté plus en détail ce passage dans «La chute de la dynastie Romanov, de E. Choub à C. Marker», Matériaux pour l'histoire de notre temps, $\mathrm{n}^{\circ} 89-90$, janvier-juin 2008, pp. 20-29.

8 D. Vertov, «Contre la phrase de gauche», inédit, écrit le 5 juillet I927, en réponse à l'article d'Ossip Brik «Protiv janrovykh kartinok» [Contre des images du genre] (Kino, ${ }^{\circ}$ 27, juillet I927).

9 Ces critiques visent sans doute, en partie du moins, Vertov qui était en butte aux attaques des membres du LEF après qu'il les eut, lui-même, récusés, comme il avait récusé Aleksei Gan et Esfir Choub.

10 L. Kouléchov, "L'Ecran aujourd'hui », dans L'Art du cinéma et autres écrits (I9I7-I934), Lausanne, L'Age d'Homme, I990, pp. I40-I42. Ce commentaire consonne avec les préconisations de Chklovski demandant de ne pas faire violence au matériau (voir supra). 
11 «Dokumentarischer und küntlerische Film», Film-Kurier, I $^{\text {er }}$ juin 1929 (repris dans Anton Kaes, Nicholas Baer, Michael Cowan, éd., The Promise of Cinema: German Film Theory, I907-I933, Oakland, University of California Press, 20I6, pp. I03-IO4).
Cette démarche fit des émules dans le mouvement du cinéma prolétarien des pays capitalistes là où il s'est développé (Grande-Bretagne, Etats-Unis, Allemagne). Willi Münzenberg fit d'ailleurs venir Choub en Allemagne en I929 pour apprendre les techniques du re-montage aux cinéastes militants allemands. Il en résulta un film intitulé Canons ou tracteurs (Segognja, I930). Il y eut même une controverse intéressante à relever entre deux "monteurs» allemands et Vertov. Mandatés par la Prometheus pour confectionner des films d'agitation révolutionnaire sur le thème de l'exploitation des ouvriers (cadences, taylorisme), Albrecht Viktor Blum et Leo Lania (ce dernier plutôt scénariste-commentateur) puisèrent en effet dans certains films de Vertov, lequel protesta de voir ses images détournées lors de son voyage en Allemagne. Pourtant Blum, qui travailla avec Erwin Piscator (où le cinéma était utilisé lors des mises en scène) et collabora à l'adaptation cinématographique de Hoppla, wir leben! (I927) d'Ernst Toller en recherchant des documents d'archives filmiques, monta pour la diffusion militante en Allemagne Le Document de Shangaï (1928) de Bliokh sur les luttes sociales en Chine, était un féru de la doctrine du «fait» contre la fiction et de la supériorité du documentaire où «les objets en eux-mêmes, dans leur vérité, atteignent leur pleine valeur» sans qu'il faille adopter une perspective artistique susceptible de les altérer ${ }^{11}$. Le dialogue avec Vertov n'eut pas lieu, ce dernier revendiquant la paternité des images qui provenaient de La Onzième Année et que Blum et Lania avaient utilisées dans leur film Im Schatten der Maschine (A l'ombre de la machine, 1928). En dehors de la question de l'auteur, cette controverse révélait cependant un autre problème: en tant que militants communistes, Blum et Lania avaient pour tâche de dénoncer l'asservissement de l'homme à la machine, l'exploitation capitaliste faisant bon marché des corps des ouvriers voués aux cadences, aux accidents, et ils mettaient l'accent sur les mutilations que les machines généraient. De son côté, Vertov exaltait au contraire ces mêmes machines mises au service de la construction du socialisme et il faisait l'impasse sur les contradictions que pouvait susciter le machinisme dans une société censée libérer les travailleurs. Sa perspective productiviste l'amenait au contraire à mécaniser le mouvement humain comme on le voit dans L'Homme à la caméra où la machine-cinéma qui s'inscrit dans la même topique que la machine industrielle accélère les gestes automatiques de l'ouvrière confectionnant des paquets de cigarettes. 
La systématisation du principe selon lequel il ne s'agissait pas tant de tourner des images soi-même que d'organiser des images existantes anima des débats passionnants au sein du LEF à la fin des années 1920 au moment où des écrivains, théoriciens, sociologues, dramaturges et cinéastes comme Tretiakov, Chklovski, Choub, Arvatov, etc. débattent de ces questions en tentant d'articuler factualité des images et discursivité du montage et discutant des gradients de falsifiabilité des images (matériau filmique) par rapport au matériau, au fait enregistré. Les Léfistes proposaient alors un programme du tout-documentaire, du tout-factuel (renchérissant sur le ciné-fait de Vertov qu'ils jugeaient devenu trop poétique, détaché de son référent) comme ligne du parti bolchévik. Celui-ci convoqua, en effet, en I928 la première conférence sur le cinéma destinée à réfléchir aux tâches à lui attribuer, à tirer le bilan de quelques années de films tiraillés entre des directions très différentes les unes des autres (des agit-films, sketchs mobilisateurs, aux restitutions littérales des événements ou aux films éducatifs visant à l'hygiène ou au simple mode d'emploi - d'une machine par exemple -, aux fictions reprenant des schémas narratifs d'aventure ou policiers pour capter l'attention du public, aux films épiques sans personnages individués, voire au cinéma «intellectuel» abstrait susceptible de développer des notions tirées du Capital, etc.). Il n'en fut évidemment rien et le choix politique inclina vers un cinéma de la représentation et de l'illustration qui se développera ultérieurement dans le cadre du réalisme-socialiste. L'un des rares exemples de film «léfiste» - si l'on écarte ceux d’Alekséi Gan qui sont antérieurs à cette réflexion - aura été un film de montage intitulé L'OEil de verre (I930, réalisation Vitali Jemtchoujny et Lili Brik) et quelques courts métrages de Jemtchoujny qui ne furent que peu convaincants. Cependant les films de montage et l'utilisation d'images d'actualités dans les films ne cessa pas en URSS, en particulier durant la Deuxième Guerre mondiale et peu après elle (films-sommes de Dovjenko, Karmen, Ioutkévitch, etc. sur la libération du territoire soviétique). Le plein retour à cette pratique est attaché au Fascisme ordinaire (1964) de Mikhaïl Romm, contemporain d'autres réalisations dans le monde s’inscrivant dans ce genre ( $L e$ Temps du ghetto, I961, Mourir à Madrid, 1963, Frédéric Rossif), qui s'était cependant quelque peu compromis et décrédibilisé durant la guerre en se mettant au service de démonstrations faisant fi du sens originel des matériaux dans un camp comme dans l'autre (la série Pourquoi nous 
12 A la fin des années i960, la production cinématographique soviétique annuelle est de i5o longs métrages de fiction, 40 longs métrages documentaires, ıoo films d'animation, iooo courts métrages d'actualités ou documentaires. combattons, I942-1945, sous la direction de Capra, les montages tendancieux des nazis, Der erwige Jude, I940, etc.).

On retiendra pour finir la permanence d'un intérêt pour les matériaux d'actualités ou documentaires de la part des cinéastes soviétiques qui soit se vouent complètement au documentaire (Péléchian), soit intègrent souvent des passages relevant du «film de montage» au sein de fictions, en particulier historiques: ainsi Tarkovski dans Le Miroir (I975) puise-t-il dans les bandes documentaires tournées pendant la Deuxième Guerre mondiale, tout comme Andréi Mikhalkov-Kontchalovski dans Sibériade (I979), Elem Klimov dans Va et regarde (ou Requiem pour un massacre, I985) et Alexandre Sokourov dans la plupart de ses films ( $L a$ Voix solitaire de l'homme, 1978, Insensibilité chagrine, 1983, Le Jour de l'Eclipse, 1988, et plus récemment Francofonia, 2015).

Le cas de ce dernier cinéaste est cependant caractéristique, plus encore que les autres, de cette intrication entre documentaire et fiction qui perdure dans le cinéma soviétique en raison de l'importance accordée aux images d'actualités, aux films scientifiques, de vulgarisation, aux documentaires sur des régions, des pratiques, des réalisations, etc. dans l'URSS des années I960-1990 12. Le VGIK, l'école de cinéma de Moscou, comporte des sections documentaires aussi importantes que celles vouées à la mise en scène, et le passage par le documentaire semble un chemin pédagogique courant, comme dans d'autres pays socialistes de l'époque (en Pologne - songeons aux nombreux documentaires de Munk - comme en Hongrie - Jancsó - ou Tchécoslovaquie - Chytilova). Sokourov a d'ailleurs intégré le VGIK après une première expérience pratique à la télévision de Gorki et il fut admis dans la section «Naoutchnopopouliarnoe kino» (le cinéma de vulgarisation scientifique) que dirigeait le cinéaste Alexandre Zgouridi, spécialisé dans les films de ce type sur la vie des animaux notamment. Sans compter les insertions de passages documentaires dans les longs métrages de fiction, sa filmographie comporte actuellement plus de documentaires que de fiction sans que la chronologie laisse apparaître un éloignement de ce genre au cours des années. Parmi ces documentaires, plusieurs films peuvent être rattachés au «film de montage», en particulier la Sonate pour Hitler (Sonata dlia Hitlera, 1987). Ce film est fait de plans courts, de fragments, d'images d'actualités et joue de figures associatives relevant de l'attraction, du photo-montage telle la répétition de certains plans - notamment d'un 
ouvrier aveugle assemblant des munitions en usine, figure de l'enchấnement mortifère des événements. D’autres raccords de plans sont plus classiques voire convenus comme cet ouvrier d'une usine d'armement essayant la visée d'un fusil auquel succède un plan sur un cadavre, ou un plan de tirs à l'arc dans la forêt (Goering) reliés à une «cible» arbitraire (plans empruntés au Fascisme ordinaire de Romm). Mais à cette dimension d'«agit-film», de "ciné-tract», s'ajoute une deuxième ligne plus profonde dans l'articulation des images entre elles et des images et des sons. Elle relève de cette attention au signifiant, une sorte de forme guettée par la monstruosité et la barbarie puisqu'elle peut indiquer la quasi-harmonie d'une gestuelle criminelle (celle de Hitler au micro), l'unanimité des mains levées des foules au passage du Führer. Sokourov engage cette dangereuse tentation, que la musique de Bach aggrave encore, puis l'interrompt brusquement, la brise ${ }^{13}$. Outre d'autres mains (celles des travailleurs à leurs machines), ce sont alors des images insolites ou macabres, un son grinçant, insistant dans son incertitude, c'est la déroute d'un sens obvie, c'est l'avènement d'un sens obtus. Des vieilles berlinoises dans la ville détruite, un officier nazi pendu, des soldats allemands lutinant des jeunes filles en costume régional, un évêque mêlé à l'armée ou Hitler tapotant la joue d'un petite fille. Ces images sont parfois des clichés, on les a vues déferler en masse dans les documentaires télévisés, on les a croisées dans Germania ano zero (1948, Rossellini), mais alors elles étaient liées par le récit, la démonstration. Là elles offrent une sorte d'opacité insulaire, elles se heurtent, elles ne se relient pas les unes aux autres, elles présentent au mieux des contrastes et des exclusions réciproques, elles suscitent l'effroi (plan récurrent d'une jeune femme au regard angoissé).

On pourrait évoquer pour finir le Blokada (2006) de Serguéi Loznitsa bien que ce réalisateur né en i964 n’entre au VGIK qu'en I99I où il passe sept ans. Quoique ayant étudié dans la période dite "post-soviétique», il est cependant, quant à sa formation, un produit typique de l'URSS des années I980-I990: né en Biélorussie, il fait d'abord des études de mathématiques et d’ingéniérie à Kiev, en Ukraine, et devient cybernéticien, avant de se rendre à Moscou et d'entreprendre des études de cinéma dans la classe de la réalisatrice géorgienne Nana Djordjadze. Il entre ensuite au studio des films documentaires de Leningrad (avant d'émigrer en Allemagne).
13 Sokourov dit avoir eu l'idée de réaliser ce film dans les années i960 après avoir vu Le Fascisme ordinaire de Romm. On peut rapprocher l'usage qu'il fait de la musique de la proposition démonstrative qu'avait faite Istvan Szabo dans un film d'école où il accompagnait la même suite d'images de la guerre de I939-I945 avec trois musiques différentes donnant aux documents dans chaque cas une signification différente. 
14 Loznitsa a précisé que pour ce film de 52 minutes il avait bénéficié de 4 heures de matériel environ - ce qui paraît fort peu et quasi impossible sauf à recourir à des films ayant déjà opéré dans des synthèses sur le sujet un premier montage et organisé une première cohérence narrative et chronologique.
Blokada retrace sans commentaire les 900 jours du siège de Leningrad par les troupes allemandes et finlandaises (8 septembre $1944^{\mathrm{I}-27}$ janvier I944) qui valut à la ville i 800 ooo morts, dont un million de civils, dus à la faim, aux bombardements et aux combats. Bénéficiant des prises de vue d'actualités très cohérentes qu'il a pu sélectionner dans l'énorme corpus des actualités militaire soviétiques ${ }^{14}$, Loznitsa assemble un certain nombre d'événements quotidiens qui, dans chacune des parties de son film, forment une unité en déroulant une chronologie et le passage des saisons. En été I94I, on assiste à la mise en place des moyens d'observation aérienne de l'armée, à la mise en alerte des canonniers de la défense anti-aérienne, dans une ville qui vaque alors normalement à ses activités (passants, tramways, automobiles, magasins, etc., activités bruitées pour en accentuer la place dans la représentation). Puis on introduit des ballons de barrage destinés à empêcher les vols d'avions ennemis, rangés sur un terrain gardé, placés çà et là dans la ville; et on suit le transport de l'un d'eux dans une rue qui se poursuit avec une enfilade de semblables engins que l'on conduit sous des arbres avant qu'ils ne prennent leur envol, série de gros poissons au-dessus de la ville. Après une première césure (écran noir), apparaissent des plans où l'on suit un cortège de prisonniers allemands entourés de soldats qui défilent dans les rues, parmi les maisons en ruine. Les passants, très nombreux sur les trottoirs et sur la chaussée, les regardent et une femme vient brièvement insulter certains et cracher en leur direction. Ils sont conduits jusqu'à des quartiers semi-ruraux et disparaissent. Cette prolepse est la seule intervention bouleversant la chronologie historique des trois années de siège. On revient ensuite à cette vie «normale» d'une ville qui se prépare à la guerre sans pour autant cesser ses activités coutumières. Des planches dressées le long de façades, un tram qui remorque un wagon de charbon sont les seuls indices instillant la tension qui s'empare de la cité. Et c'est soudain une alerte précipitant les passants en direction des abris. S’ensuit l'immobilité des trams et des autobus. Avec la nuit et les projecteurs de la DCA surviennent les premiers bombardements et interventions des pompiers et des brigades de volontaires; on s'attarde sur l'incendie d'une bibliothèque de plusieurs étages dont les rayonnages de livres en feu tombent sous les lances d'incendie sur le trottoir d'où on les évacue. Ce sont ensuite les ruines, les évacuations des habitants sinistrés et les premiers morts - dont une 
petite fille sur un escalier (qu'on reverra dans le montage d'archives de Sokourov de Francofonia). Les chevaux de frise, casemates, régiments et véhicules blindés prennent progressivement l'avantage sur les habitants dont les activités habituelles sont maintenant suspendues. Les affiches mobilisatrices se multiplient sur les murs, les caricatures antifascistes, les appels à l'entr'aide et à la production d'armes pour la défense de la ville. La ville est maintenant couverte de neige et les canaux de glace, le ciel chargé de continuelles fumées noires, et les habitants viennent puiser de l'eau dans des tranchées. Les cadavres se multiplient le long des rues; on les transporte sur des luges et on les empile dans des fosses creusées dans la glace. Finalement, après des coups de canons tirés dans la nuit par le croiseur Aurore dans la baie de Leningrad, ce sont les mines réjouies de la foule fêtant la victoire, la fin du siège sous les feux d'artifice (en janvier i944). Les derniers plans - renouant avec ceux des prisonniers allemands conduits dans la ville placés au début du film nous font enfin assister à la pendaison collective d'un certain nombre de condamnés. La foule est alors énorme, elle forme une houle autour des gibets ${ }^{15}$.

Loznitsa inscrit son film à la fois dans la filiation et en opposition à un grand nombre de films consacrés au blocus de Leningrad, le plus long et le plus meurtrier qu'une ville ait eu à subir à l'époque contemporaine. Il se refuse à tout commentaire et laisse le spectateur en face de scènes quotidiennes ou spectaculaires, d'événements douloureux, tragiques et ne montre aucune scène de combat. A peine tire-t-on sur les avions allemands venus bombarder la ville. En revanche on se trouve du côté des habitants, des victimes, y compris les militaires qui assurent la défense du lieu, et on met l'accent sur les activités de secours et sur la survie des habitants dont le plan final dit ambigument qu'il a vaincu et s'est vengé.

Très économique dans ses moyens, très rigoureux dans ses choix d'images, ce film de montage, s'il est sans parole, est cependant sonorisé. C’est sans doute le premier problème qu'il pose. Dès le premier plan qui montre la Néva et les silhouettes de bâtiments religieux exactement reflétés dans l'eau, on a le bruit du courant, puis les pas de soldats qui défilent, des vrombissements d'avions, de moteurs de camions, de passages de tramways et de leur avertisseur, de lointains klaxons, des cliquetis d'appareils, de manivelles, des cris indistincts, des aboiements,
15 Des plans qui appartiennent à une période ultérieure puisque, selon le carton final, il s'agit de l'exécution des jugements pour crimes de guerre à l'endroit de prisonniers allemands datant du 5 janvier 1946. 
16 La vitesse de déplacement des passants dans plusieurs plans «bruités» de manière détaillée atteste de la mutité d’origine qui est la leur.

17 Le générique de fin énumère les noms de 28 opérateurs.

18 Il y en eut en effet un certain nombre (l'un dû à Roman Karmen), dont quatre sont signalés dans le générique de fin comme ayant été mis à contribution pour ce film. des sirènes, des crépitements d'incendie. Ce choix altère quelque peu la rigueur de la démarche et en accuse un certain esthétisme - qu’on trouve aussi dans le choix de ces images «muettes» - en même temps qu'il assure un «effet de réel» illusoire et finalement rassurant. On obtient ainsi parfois des effets quasi anecdotiques. On fait, par exemple, «sonner» le tram quand il dépasse des soldats transportant un ballon de barrage le long d'une rue: pure recherche du vraisemblable. Ailleurs c'est le "gros plan» sonore du passage d'un camion off qui vient faire contrepoint à la silhouette d'un guetteur. La sirène ajoutée aux plans de passants courant - sans doute vers des abris - vient expliciter la cause de cette agitation mais en atténue du coup la violence et l'étrangeté. On entend à l'occasion des cris ou des plaintes, des sirènes de pompiers ou d'ambulance montés sur des plans qui étaient de toute évidence muets ${ }^{16}$ (les caméras sonores étaient extrêmement rares en URSS à cette époque-là et l'usage n'était pas d'enregistrer les sons «en direct» pour les actualités - en dépit des préconisations de Vertov...).

Par ailleurs, on s'interroge dans ce film comme dans la plupart des films de montage sur la part quy prennent les différents agents ayant contribué à la production des images et sur le statut des différentes instances du filmique: le travail de prise de vue des opérateurs, le soin des cadrages et le «suivi» des événements en question sont remarquables. Cette conscience de couvrir un fait si bénin soit-il (comme l'acheminement des ballons), d'en faire le tour (sauvetage de livres ou de documents évacués d'un bâtiment bombardé) sert manifestement Loznitsa. Eut-il à assembler, dans telle ou telle séquence, des prises de vue d'opérateurs différents ou d'un seul, ou d'une seule équipe ${ }^{17} \mathrm{~A}-\mathrm{t}-\mathrm{il}$ repris des séquences déjà montées ainsi dans un «journal d'actualités» de l'époque ou dans un film de synthèse sur le blocus réalisé par la suite? ${ }^{18}$ Quelle place les opérateurs et les premiers monteurs octroyèrent-ils à un certain nombre d'effets plastiques qui deviennent chez Loznitsa quasi formalistes - comme ces ombres qui se projettent sur la surface convexe d'un ballon que mettent en place des soldates sous des arbres: en s'approchant, face à la caméra, cette baudruche oblongue grossit et développe une sorte de mouvement stroboscopique fascinant. Enfin quelle est la part, dans le montage même de plan à plan, qui lui revient en propre? Par exemple, on enchaîne le plan d'un observateur scrutant le ciel avec celui de canonniers pointant leurs armes vers le haut. 
Ce sont des questions que seuls les spécialistes des documentaires de guerre sont en position de nous apporter - par exemple en nous renseignant sur les protocoles de prises de vue que doivent ou non respecter les opérateurs, sur les figures de montage récurrentes dans les actualités, etc. -, non pour diminuer la qualité de ce film mais pour en préciser la teneur et l'expliquer mieux, comme pour mesurer les effets de distance recherchées voire les penchants esthétisants qui l’affectent. 